\title{
LA FORMATION PROFESSIONNELLE COMME EDUCATION DU REGARD AU TRAVAIL
}

\author{
Guy Jobert
}

\section{Introduction}

Chacun sait qu'un «vrai » professionnel est celui «qui a l'œil », qui voit vite ce qui ne va pas, ce qu'il faut faire et comment le faire, sans délai et sans erreurs. On dit d'un chanteur ou d'un instrumentiste "qu'il a une bonne oreille » pour signifier la même chose. Il y a dans les cuisines ceux dont le goût et l'odorat sont sans pareil, comme on dira d'un œnologue «qu'il a le nez». Dans les menuiseries certains touchent le bois et savent d'emblée à quoi ils ont affaire et ce qu'ils peuvent en faire. Quel que soit le sens mobilisé au travail : regard, odorat, toucher, kinesthésie, il semblerait que, dans le langage courant, les perceptions corporelles multiples privilégient le regard de « celui qui a l'œil ».

Si c'est vraiment dans cette compétence sensorielle multimodale, dans cet engagement du corps sensible que se concentre l'expertise du professionnel, on comprend l'intérêt que peuvent porter les formateurs et les enseignants techniques à la façon dont se fabrique ce regard expert, concentré de l'ensemble des perceptions sensorielles qui assure la réussite de l'action, donne accès au statut de professionnel et le distingue du profane qui, lui, ne voit rien, ne sent pas, n'entend pas, ou lorsqu'il voit, ne comprend pas, ou s'il comprend quelque-chose, ne sait que faire de ce qu'il a compris.

Ces constats portent à émettre une hypothèse forte que ce texte argumentera : la formation professionnelle pourrait être considérée comme une modalité particulière du développement humain, de la même façon que le philosophe Yves Schwartz (1988) situe la 
compétence au travail comme une modalité particulière d'une plus large « compétence à vivre » anthropologique.

En décrivant en ethnologue du travail, et non en pédagogue, en aval donc de toute ingénierie de formation, certains usages, fonctions, modalités d'exercice de ce regard intelligent et, à bien des égards énigmatique, ce texte s'appuie sur un matériel empirique recueilli à l'occasion de recherches-interventions menées par l'auteur au fil des années (empirie sans laquelle l'anthropologie devient métaphysique), dans différents milieux de travail (production cimentière, nucléaire, agroalimentaire, mécanique). Ce texte se veut au plan pratique, une aide à la définition des «états-buts» que le formateur peut proposer aux apprenants et au plan scientifique, une première contribution à une anthropologie de la figure postcartésienne de "l'homme capable», selon la formule de Paul Ricoeur (1998). C'est ainsi que sera proposée une catégorisation des regards au travail que nous avons appelés regard herméneute, regard didactique et mimétique, et regard praxique.

\section{Le regard herméneute}

\subsection{Regarder la matière : fabriquer du ciment}

Produire du ciment consiste à broyer un mélange de calcaire et d'argile avant de le cuire à 1450 degrés. Le four dans lequel s'opère cette cuisson est au cœur du procès : c'est là que se joue la réussite ou l'échec de la production de ciments de qualités variables selon leur destination (un mur de maison en plaine ou un pilier de pont immergé en eau salée ne requièrent pas les mêmes qualités de produit). Le four se présente comme un long cylindre de métal incliné, tournant en permanence, alimenté en partie basse par un brûleur de fort diamètre qui envoie une flamme d'une dizaine de mètres à l'intérieur du four et de la matière pulvérulente en mouvement lent. La chaleur et la longueur de la flamme, ainsi que la vitesse de rotation du four, sont les variables sensibles qu'il convient d'ajuster en fonction des singularités mentionnées plus haut.

L'entreprise, premier producteur mondial, a fait appel à une équipe de chercheurs intervenants pour l'assister dans son projet 
d'automatisation de l'usine, notamment pour étudier l'activité des conducteurs du four qui devront, à l'avenir, rester capables de piloter manuellement l'installation en cas de défaillance des automatismes. Étrangement, mais pas extraordinairement, le contenu de l'activité des conducteurs du four semble mal connu, tant des ingénieurs que de la maîtrise de premier niveau. En conduite manuelle, le four doit être contrôlé et éventuellement réglé régulièrement par des opérateurs qui se succèdent ; c'est une aubaine pour les chercheurs qui peuvent ainsi observer les mêmes opérations effectuées par divers opérateurs.

Première observation: l'opérateur s'approche de la base du four proche du brûleur, s'empare d'un masque semblable à celui d'un soudeur à l'arc, soulève une trappe par laquelle on voit l'intérieur du four, flamme et matière. Il marque un temps d'arrêt de quelques secondes. Plusieurs fois, son corps s'avance plus près du cylindre et du brûleur pendant deux ou trois secondes. Après un dernier regard, genoux légèrement fléchis, il referme la trappe, raccroche son masque et ouvre une armoire dans laquelle se trouvent quelques instruments de réglage qu'il effleure rapidement (on aura compris qu'au moment de l'observation, l'automatisation complète n'est pas effective). Après quoi, l'opérateur s'éloigne de son four.

Deuxième observation: le deuxième opérateur procède, comme son prédécesseur, à quelques petites différences près : le temps d'observation des entrailles de la machine est plus long; l'opérateur marque plus que son collègue un léger basculement du corps d'arrière en avant et, surtout, son réglage exécuté et l'armoire fermée, il se retourne avec une certaine brusquerie vers la trappe pour regarder à nouveau la cuisson, oubliant au passage de se munir du masque à la vitre bleutée. C'est alors seulement qu'il s'éloigne du four.

Lors des échanges qui suivent les observations, tout ce que les chercheurs avaient cru comprendre de ce qu'ils avaient observé a été entériné par les opérateurs, en particulier s'agissant du retour prudentiel du second opérateur. Les opérateurs ajoutent que leur métier consiste à regarder pour "savoir comment ça se passe » au cœur de la fournaise inaccessible, mais qu'ils se font « aider» (sic) par leur corps, placé plus ou moins près du cylindre pour en ressentir la chaleur et les vibrations, ou encore par l'ouïe : " moi, quand j'entre dans le local, j'entends tout de suite si le brûleur c'est bon, pas besoin 
d'aller y voir ». Au cours des échanges, les agents disent aussi que le premier opérateur observé est considéré comme un très grand professionnel et, pour souligner la part d'énigme que comporte sa compétence, les agents avouent: " même nous on comprend pas toujours comment il fait. C'est comme vous; vous savez, vous n'avez pas tout compris; en réalité c'est très compliqué. C'est sans doute pour ça qu'ils sont allés vous chercher; ils ont la trouille de passer en tout automatique ».

\subsection{Regarder des artefacts : les systèmes productifs automatisés}

$\mathrm{Au}$ fil des innovations technologiques, les systèmes techniques de production sont passés par trois étapes qui, toutes, ont affecté le regard mobilisé dans l'action. Un premier temps a été celui où le travailleur était dans un rapport corporel direct avec la matière à transformer, le produit de son travail et l'outil qui prolongeait son corps. C'est le modèle de l'artisanat et celui de la manufacture. Avec l'arrivée de machines de plus en plus autonomes, l'opérateur a été séparé de la matière en transformation. Avec l'automatisation des processus productifs, la matière est à distance, l'outil et son fonctionnement sont des boîtes noires inaccessibles, Le procès luimême, dématérialisé, est devenu invisible. Le travailleur n'a même plus à surveiller et à régler sa machine. Les automatismes s'autocontrôlent en continu et interviennent de leur propre initiative pour réguler l'installation en cas de dérive hors des limites des tolérances définies en amont par les concepteurs des équipements.

Après la disparition du regard sur la matière, c'est la totalité des éléments constitutifs ou contributifs de l'activité humaine qui sont devenus des abstractions, des transferts invisibles d'informations montantes ou descendantes ${ }^{1}$. L'opérateur vit dans un monde sans bruits ni odeurs. Le corps sensible de l'opérateur, sa métis, cette intelligence du corps qui mobilise les sens et suscite une intelligence «rusée » adaptative plutôt que l'application de procédures face à l'incertitude et l'imprévisibilité des situations, n'est plus sollicitée pour mettre en relation les hommes, les machines et l'environnement,

\footnotetext{
${ }^{1}$ Sur la question de l'informatisation des activités de travail, on pourra consulter l'ouvrage d'Yves Clot (1995).
} 
à l'exception toutefois du regard, seul sens capable de percevoir les représentations symbolisées du système technique automatisé.

\subsubsection{Produire de l'électricité}

Parmi les unités de production automatisées, les centrales nucléaires apparaissent comme des archétypes. Le lecteur est convié à observer le regard herméneute des opérateurs humains estimés encore nécessaires, en petit nombre, à la maîtrise d'installations hypercomplexes à haut risque. Concrètement, pour chaque réacteur, des opérateurs (pupitreurs) sont rassemblés dans une salle de commande centralisée, souvent dénommée «bloc». Ce local est saturé d'écrans, d'instruments en tout genre qui couvrent les murs et encombrent l'espace. On y trouve une quantité difficilement imaginable d'indicateurs qui informent les opérateurs des mesures prises en continu par des centaines de capteurs répartis dans l'installation physique. L'installation s'autorégule, mais dans l'hypothèse d'un écart que la machine n'aurait pas corrigé elle-même, des alarmes optiques et acoustiques apparaissent sur les pupitres pour requérir une intervention humaine ${ }^{2}$.

Deux pupitreurs ont été observés par deux chercheurs sur des séquences courtes, non filmées.

Première observation : l'opérateur est en début de poste et, comme le veut la procédure, «prend le pouls » de sa «tranche» (nom donné au réacteur) qu'il a quittée la veille au soir; il doit consulter trois écrans proches qui fournissent des mesures de paramètres de fonctionnement essentiels (il est question de température, de pression, de disponibilité d'un équipement majeur). L'opérateur entre, longe lentement le long mur couvert de tableaux, s'arrête à un endroit à l'évidence bien repéré; un léger lever de tête, corps parfaitement immobile, le regard, fixe, est porté sur trois écrans quelques secondes, léger recul du corps, deux secondes d'attente, et l'opérateur termine sa marche pour rejoindre ses collègues.

Deuxième observation: tout se déroule à l'identique à quelques détails près : le temps d'observation du pupitre est un peu plus long; il s'accompagne de légers mouvements latéraux de la tête ;

${ }^{2}$ Cette recherche-intervention a fait l'objet d'un ouvrage (Jobert, 2014). 
le corps, comme celui de son collègue, s'écarte du pupitre, mais l'opérateur reporte à nouveau son regard vers les indicateurs. Après un hochement de tête, il va à son tour rejoindre ses collègues.

La séquence d'observation est suivie d'une réunion entre chercheurs et opérateurs. Le ton en est assez vif ; les agents expliquent que s'ils n'avaient pas eu avec eux l'un des chercheurs connu pour avoir mené plusieurs recherches dans l'entreprise et construit des relations cordiales et de confiance, ils n'auraient pas accepté l'observation faute d'être assurés qu'elle ne pouvait pas leur nuire. Pressés de questions, ils justifient la différence de tempo entre les deux opérateurs: elle s'explique par le fait qu'il ne s'agit pas de regarder successivement, et donc séparément chacun des écrans, mais de les saisir ensemble, d'un même regard, et d'interpréter les informations les unes par rapport aux autres car les phénomènes symbolisés au tableau sont eux-mêmes, sur le terrain, en interrelation dynamique. L'activité cognitive attachée au regard se déploie sur plusieurs plans : par un traitement in situ des informations fournies par les synoptiques, et par une mise en relation mentale entre phénomènes symbolisés à distance et phénomènes réels de l'installation physique.

C'est ainsi que l'un des pupitreurs dira en réunion de "débriefing»: "tu comprends, c'est ça le coup d'œil qui fait la différence. Lire chaque écran, tout le monde l'apprend en formation, mais pour tout comprendre en même temps, il faut avoir dans sa tête comment c'est en physique, avoir des images quoi!». C'est cette capacité interprétative que nous désignons comme herméneutique du regard, qui fait la différence entre qualification acquise et compétence exercée. Dans les termes de la didactique professionnelle, on dira que l'opérateur interprète l'état du système à partir d'un modèle opératif, ou d'une représentation fonctionnelle, qu'il a construit par son expérience et qui dirige à la fois sa recherche d'informations, ses perceptions et ses raisonnements à l'issue desquels il élabore une compréhension de la situation. Pierre Pastré (2011), en didactique professionnelle, montre la différence entre un modèle cognitif du fonctionnement d'un système, qui peut être appris en formation, et un modèle opératif, qui réélabore et enrichit le modèle cognitif par l'expérience des situations et des actions, et par l'usage du corps et des perceptions. 
Au fil des échanges, les pupitreurs décrivent leurs « ficelles de métier», sortes de «béquilles cognitives» au service du regard herméneutique: "On se fait dans la tête un schéma de la bonne position des aiguilles des traceurs"; " c'est dedans ou ce n'est pas dans mon schéma. C'est ça qui nous guide, on gagne du temps et c'est plus sûr ».

Cette remarque sur l'installation absente mais «que l'on a dans la tête » a également conduit les chercheurs à la compréhension, ultérieurement approfondie et généralisable à la conduite de tous les systèmes complexes automatisés, et à la thèse suivante : ce ne sont pas les opérateurs qui sont dans l'installation, c'est l'installation qui est incorporée en eux. Lorsqu'ils regardent les écrans, les pupitreurs ne mettent pas en rapport la symbolique abstraite des écrans avec des objets lointains du monde extérieur mais avec des images intériorisées de ces objets du monde invisible. Ce retour d'un «corps contenant» permet d'établir un pont entre le réel de la production et l'abstraction imposée par les automatismes. Ces opérateurs doivent agir sur ce qui n'est pas là.

Á l'appui de la thèse d'une installation incorporée, nous avons recueilli un abondant matériel empirique, parfois surprenant, mais nous avons relevé également, comme nous l'illustrons ci-dessous, qu'il peut y avoir des risques à piloter un équipement qu'on a " dans son ventre » plutôt que celui du monde objectif. Cette incorporation d'un objet du monde extérieur fait penser à ce que Jean Piaget a décrit comme processus d'incorporation/assimilation du premier stade de développement de l'enfant. Ce qui est très particulier et intéressant dans le cas des travailleurs de l'abstrait que sont les pupitreurs des salles de commande, c'est que la loi de permanence de l'objet au fondement du rapport au réel de l'enfant, et qui se prolongera par la capacité de symbolisation, s'applique non pas à un objet du monde extérieur qui continue à exister hors du regard du sujet mais à un objet du monde intérieur, celui qui a été incorporé par l'opérateur et qui va continuer à exister sous forme d'une représentation mentale.

Nous avons pu, par nos observations, montrer qu'un opérateur, lorsqu'il est en congé, continue à entretenir en lui la vie de son réacteur et que lorsqu'il retrouve celui-ci, il reprend son pilotage dans la situation où il l'avait laissée, dans l'état où se trouve en lui son 
réacteur intériorisé, ce qui n'est pas sans conséquences possiblement fâcheuses. L'analyse approfondie d'un incident, très sérieux mais sans conséquences, que nous avons pu réaliser dans une centrale nucléaire a montré que l'opérateur fautif avait réglé sa conduite sur l'état incorporé de son réacteur qu'il avait quitté la veille après son poste de l'après-midi en négligeant de regarder des informations exogènes ("pour moi, c'était clair, j'étais au niveau 5 de puissance», ne cessait-il de répéter au désespoir, pendant le déroulement de l'incident). Dans sa conscience, son réacteur (interne) était à ce niveau. La chose était tellement certaine pour lui que le passage par le cadran a été négligé dans le feu de l'action.

\subsubsection{Piloter un avion}

Un autre exemple de regard professionnel porté sur des artefacts est fourni par le pilotage d'avions de ligne. La « machine»c'est ainsi que les pilotes nomment leur aéroplane - est un condensé sans équivalent de calculateurs, d'automates, d'asservissements... Depuis bien longtemps, les pilotes de ligne ont perdu tout contact corporel avec le monde naturel par leurs sens (à la différence des pilotes de chasse qui disent volontiers que leur avion se pilote « avec ses fesses »). Avec les dernières générations d'avions de transport, les pilotes ont perdu tout contact non seulement avec le milieu naturel mais avec la machine elle-même dont toutes les commandes sont asservies ou commandées directement par des ordinateurs. L'autonomie des automatismes est telle que le rôle des pilotes consiste non plus à surveiller la machine pour en corriger les approximations mais, dans bien des circonstances, à tenter de comprendre ce qu'elle est en train de faire, ce qu'elle a décidé de faire. Le retour de la cognition et de l'action humaine consiste ultérieurement, si l'avion vole encore, à chercher à comprendre ex post, ce que la machine a perçu, diagnostiqué, décidé et exécuté d'elle-même.

Lorsqu'un pilote amorce sa manœuvre d'approche, en pilotage dit «tête baissée » (c'est-à-dire aux instruments), il s'en remet à la machine qui sait faire mais doit cependant «avoir à l'œil» trois paramètres que ni lui ni sa machine ne doivent transgresser : sa vitesse, son taux de descente et son altitude. Comme le pupitreur en salle de commandes, son regard n'est utile que si les trois informations 
sont saisies dans leur ensemble, dans leur interaction et dans leurs relations au monde extérieur escamoté.

Aux exemples fournis plus haut, bien d'autres pourraient s'ajouter, venant des secteurs administratifs, des services ou des métiers du soin. Pensons au regard classificatoire et anticipateur du guichetier d'un bureau de poste («quand le client vient vers mon guichet je vois à quoi je vais avoir à faire »); à celui du douanier ou du policier qui réagissent à l'écart perçu entre normalité et apparence des phénomènes; ou à celui de l'enseignant qui, d'un coup d'œil anticipe la façon dont il devra "prendre sa classe »; ou encore à l'intense activité visuelle d'une infirmière posant un cathéter, etc. Toutes ces pratiques du quotidien mettent en évidence la fonction épistémique «du regard qui sait parce qu'il interprète ce qu'il voit ». Si l'herméneutique est l'art d'interpréter ce qui est complexe ou confus pour le rendre clair et en dégager des significations qui ne se ne livrent pas d'emblée, il n'est pas illégitime de qualifier d'herméneute le regard de professionnels que l'on a vu précédemment interpréter des signes, faire parler des phénomènes complexes incompréhensibles pour le profane. II ne s'agit bien entendu pas d'herméneutique au sens originel d'herméneutique textuelle mais de sémiologie ou encore d'herméneutique de l'action.

C'est d'ailleurs presque en ces termes que le langage ordinaire parle de la mystérieuse et admirable capacité de professionnels de très haut niveau qui mêlent le voir et le savoir en un seul geste professionnel. Reprenons quelques citations: «il pouvait, juste en regardant et en croquant la pâte encore sur le tapis, dire combien il restait d'humidité à un point près" (fabrication de pâtes alimentaires); ou encore: "Ce type ne se trompait jamais; il regardait un petit coup, mettait la pièce entre ses doigts et il pouvait te dire au micron près si tu étais dedans ou dehors. Si tu avais sorti le pied à coulisse, tu l'aurais vexé » (petite mécanique de précision).

Une fois repérés empiriquement les liens entre le regarder et le comprendre, et noté leur simultanéité et leur instantanéité, il reste à rendre compte, du point de vue du fonctionnement cérébral, des liens qui peuvent exister entre l'opération sensible (regarder) et l'action sur le monde, ou dit autrement entre le sensible et le moteur, liaison que cherche à établir toute formation professionnelle : «Que vois-tu ? Que 
comprends tu? Que dois-tu faire? ». Si ces liens n'existent pas, le regard est renvoyé à une dimension seulement informative ou esthétisante. Par exemple, celle du critique d'art qui, travailleur lui aussi, sait faire parler signes et symboles, sans toutefois, en principe, avoir un projet intentionnel de transformation de l'objet qu'il regarde.

Après avoir restitué au regard sa pleine fonctionnalité sémiotique et praxéologique, il faut, pour asseoir le statut praxique d'un regard habité par l'intention d'agir sur, ou avec, l'objet regardé, examiner l'existence d'un cycle qui, physiologiquement, montrerait l'existence (visible à l'imagerie) d'un cycle reliant la perception à l'action que le sens commun dissocie.

\subsubsection{Un lien entre perception et action?}

L'empêchement à penser ensemble perception et action tient à ce qu'il est habituel de considérer comme séparés et consécutifs ce que le corps perçoit du monde intérieur et extérieur, et l'action qu'il peut exercer dans et sur ces derniers. Le sens commun tire le perceptif du côté de la passivité en lui accordant au mieux une fonction informative, propédeutique, de prélèvement d'informations sur l'environnement à seule fin d'alimenter une sorte de back-office neuronal opaque chargé de décider, d'organiser et d'ordonner l'action requise.

Cette conception dualiste est battue en brèche par les avancées des neurosciences qui ont montré que «les neurones veulent comprendre » (Varela et ali, 1993) ${ }^{3}$. Par une opération mentale unique de perception-compréhension, les neurones s'emparent de ce qu'ils prélèvent des mondes externes et internes et renvoient au système sensoriel centralisateur des informations déjà sensées qui vont alors puiser dans une mémoire disponible pour décider et guider l'agir. Cette mémoire est liée à l'histoire d'un sujet singulier, ce que l'on appelle son expérience personnelle, ajoutée à la mémoire millénaire héritée de l'évolution de l'espèce ; c'est cette riche mémoire-héritage qui guide l'action future.

\footnotetext{
3 Notons que Francisco Varela a largement promu la «Phénoménologie de la perception » de Maurice Merleau-Ponty (1945) et son refus d'opposer corps et esprit. Pour « une pratique phénoménologique », voir Depraz, Varela, et Vermersch (2011).
} 
Pour éclairer les interrogations des formateurs sur le lien entre expérience mémorisée de l'action passée, fulgurance des processus biologiques et prise de décision sensée dans l'action (question qui est au cœur de la notion de compétence située), il nous semble utile ici de citer Alain Berthoz : "Les espèces qui ont remporté l'épreuve de la sélection naturelle sont celles qui ont su gagner quelques millisecondes dans la capture d'une proie et anticiper les actions d'un prédateur, celles dont le cerveau a pu manipuler les éléments de l'environnement et choisir les meilleurs trajets de retour au gîte, mémoriser un grand nombre d'informations parmi l'expérience passée pour l'utiliser dans le feu de l'action » (1997, p. 9).

Les formateurs ont toutes raisons d'être attentifs à ce continuum qui part de la perception multi-sensorielle, passe par l'interprétation des observables signifiants (ce que nous avons appelé l'intelligence des signes) pour déboucher sur la prise de décision et l'action (ici l'activité de travail). Dans l'exemple des opérateurs de la salle de commande centralisée de la centrale électrique, on a noté que le regard porté sur les synoptiques par les opérateurs enclenchait, par automatisme acquis, une triple inférence instantanée du type: $\mathrm{Si}$ cadran 1, si cadran 2 ... (perception); Alors ... (diagnostic); Donc ... (anticipation de l'action).

Illustrons la consubstantialité de la perception et de l'action, à l'appui d'une théorie motrice de la perception, avec un exemple simple proposé par Berthoz (1997), citant Pierre Janet : " Quand nous percevons un objet, un fauteuil par exemple, nous restons debout, immobile, à le regarder en pensant ne pas faire d'action alors que le fauteuil est en train de nous dicter la façon particulière de s'asseoir dans ce fauteuil. La perception inscrit déjà en nous les caractéristiques du fauteuil qui nous dictera la manière particulière de nous y asseoir ». Dans l'exemple de la salle de commande centralisée, l'anticipation de l'action et de ses conséquences, comme composantes de la perception, signifie que les agents sont mentalement d'emblée présents dans le troisième terme de la triple inférence mentionnée précédemment, parce qu'en fait cette inférence opérationnelle ou pré-opérationnelle contient déjà les deux précédentes. Le regard est ainsi présent sur les deux versants de l'action: d'un côté, la prise en compte de la complexité et de la 
versatilité du monde; de l'autre, l'intelligence de l'action pertinente qui permettra d'obtenir le résultat recherché.

La complexité et la rapidité de l'articulation entre le déjà là cognitif (les savoirs, l'expérience), qui précède la prise de conscience, et l'action ont de quoi troubler les professionnels de l'apprentissage : les neurosciences cognitives apportent des connaissances précieuses. Le neurophysiologiste Lionel Naccache rapporte les expériences menées dans les années 80 par Benjamin Libet et en livre les conclusions: "Au moment où nous pensons prendre une décision aussi élémentaire que celle d'appuyer volontairement sur un bouton, les régions de notre cerveau impliquées dans la genèse des ordres moteurs sont, quant à elles, déjà à l'œuvre depuis plusieurs dizaines de secondes» (2006, p. 320). Il existe donc, écrit Naccache, préalablement à notre volonté consciente, une représentation mentale inconsciente; cette antériorité et ce délai des représentations mémorielles (que nous préférons appeler non conscientisées plutôt qu'inconscientes), et leur temps long de manifestation, pourra être le temps de l'introduction dans le processus de la dimension éthique de l'action, une sorte de moment phronésique rapide.

En précurseur, dès les années 60 , le psychologue américain Libet avance la notion de "véto ", c'est-à-dire la capacité d'une activité consciente à bloquer ou annuler un acte déjà engagé ; blocage possible grâce au temps restant de quelques centaines de millisecondes entre la perception subjective de la décision et l'exécution de l'acte lui-même. Plus avancés encore, les travaux récents du psychologue français Olivier Houdé (2018), disposant des moyens de l'imagerie cérébrale, ont mis en évidence le rôle du cortex préfrontal dans le « retardement », le « freinage » de l'impulsion motrice qui rend ainsi possible un "moment réflexif », et pourquoi pas délibératif, c'est-àdire axiologique. De quoi satisfaire les formateurs pour qui la compétence, et plus largement l'agir situé, doit intégrer des dimensions critiques et éthiques.

Quelles conclusions en tirer pour les sciences de l'éducation? Les développements précédents font apparaître un point essentiel pour le formateur concernant la dynamique interne de la compétence professionnelle du point de vue des capacités qu'elle requiert: la perception, notamment visuelle, mais plus largement la corporéité de 
l'agir, n'est jamais simple prise d'informations sur le monde extérieur, elle est dans le même temps interprétation et exploitation de ces informations en vue de l'action (Ochanine, 1971). Pour le formateur, « fabriquer le regard » peut donc consister à entraîner les apprenants à identifier et différencier, par une prise de conscience concomitante ou ex-post, le sensible de l'herméneutique, opération hautement formative qu'il est impossible de réaliser seul et dans le cours d'action, car le couplage perception/interprétation est rendu invisible par sa simultanéité et la fulgurance des échanges neuronaux. Le cerveau va puiser, pour interpréter ce qui est perçu, dans une jurisprudence infinie d'expériences antécédentes et de leurs conséquences. En quelque sorte, une mémoire pour prédire l'avenir. Cette construction, éminemment singulière s'insère dans un processus global de développement de la personne ; il s'agit bien d'apprentissage à visée développementale, c'est-à-dire d'acquisition de capacités d'action nouvelles.

\subsection{L'énigme de l'interprétation}

Largement argumentée par les sciences cognitives et par l'observation clinique du travail, la concaténation des opérations mentales qui s'enchaînent, depuis le regard herméneute du travailleur jusqu'à son action motrice, peut, par expérience, être aisément admise par les formateurs qui ont repéré cette composante nodale de la compétence que constitue le «coup d'œil» expert. En revanche, demeure pour eux l'énigme de la capacité herméneutique de la perception qui est au cœur de l'intelligence du regard expert et fait la différence avec le novice. Point important puisqu'il s'agit de ne pas rabattre l'expertise herméneutique du regard au travail sur des capacités innées mais d'en faire un objet et un objectif d'apprentissage. Il faut pour cela préciser en quoi consiste le repérage de ce qui fait signe, de ce qui, assez mystérieusement, alerte le professionnel sans attirer l'attention du profane ignorant ${ }^{4}$.

Nous tenterons ci-après de caractériser cette façon virtuose, et parfois d'apparence magique, propre au professionnel compétent, de voir et de connaître ce qui ne s'offre pas d'emblée à la compréhension. Pour être herméneute, le regard expert doit être clinique et le

${ }^{4}$ Sur le concept de « signe », on peut consulter l'ouvrage d'Umberto Eco (1992). 
travailleur être, par conséquent, un clinicien. Le professionnel est un clinicien. Cette affirmation, comme le vocabulaire employé, peu en usage dans le monde du travail, demandent quelques développements.

Lorsqu'un opérateur, quel que soit son secteur d'activité, observe, interprète ce qu'il voit et anticipe son action, il produit un savoir et une plus grande intelligibilité des signes qui se présentent à lui. Il ne s'agit pas d'un savoir scientifique à portée générale, mais d'un savoir local, pragmatique, du type « comprendre pour agir». Ce mode interprétatif de production de savoir portant sur un objet singulier, placé dans un contexte lui-même singulier, relève de ce que les sciences de l'homme appellent «démarche clinique» (Lhuillier, 2006).

Le moteur de ce mode particulier de production des connaissances par un individu corporellement, cognitivement, émotionnellement impliqué dans la situation qu'il cherche à comprendre en intériorité, réside dans la surprise éprouvée par celui qui cherche à comprendre au-delà des apparences (Jobert, 2006 ; Marcelli, 2006). Cette surprise, qui déstabilise, alerte et inquiète, surgit de l'écart que l'acteur découvre entre ce qu'il s'attendait à constater et s'apprêtait à trouver, et ce qu'il constate en présence du réel. L'attente peut, selon les auteurs, être appelée pré-connaissance ou préjugé, ou encore horizon d'attente, pour désigner un déjà là cognitif et émotionnel (ce que je dois m'attendre à trouver en référence à mes savoirs antécédents, ce que je dois m'attendre à éprouver affectivement et esthétiquement, en référence à ce que moi ou d'autres ont vécu en semblables circonstances).

Si la surprise, déstabilisante, naît d'un écart, cela suppose la présence de deux éléments dont la confrontation dissonante va générer l'étonnement. L'un de ces éléments, on l'a dit, est le réel perçu par le travailleur avec son corps: la matière en rotation dans le four du cimentier, le réel symbolisé du pupitre de la centrale ou du tableau de bord de l'aéronef ou, pour le médecin, ce qu'il découvre à la palpation de l'abdomen d'un patient.

L'autre élément est constitué par la masse des savoirs possédés par le clinicien à partir desquels il a construit son horizon d'attente. Ce déjà-là est épistémique; il est fait de savoirs savants et 
de savoirs expérientiels. Sans attente consciente ni présence physique active du chercheur, pas de surprise. Sans surprise, pas de questions. Sans questions, nulle nécessité et désir d'investiguer pour chercher des réponses. Les informations venues du monde extérieur ou interne sont alors indifférenciées, elles ne font pas sens et, dans bien des cas, elles ne sont pas même perçues ; ce qui peut avoir des conséquences graves dans des activités à risque. Selon Gaston Bachelard (1971), toute connaissance est réponse à une question. Combien d'ethnologues du travail ont été stupéfaits de voir des opérateurs intervenir en réaction à un évènement qu'ils n'avaient eux-mêmes pas perçus, tels que l'apparition, la disparition ou le changement d'un bruit, faute de disposer de l'horizon d'attente nécessaire à la perception sensuelle d'un écart.

Les savoirs antécédents qui précèdent la rencontre du professionnel avec une réalité singulière sont composites: connaissances scientifiques plus ou moins dégradées, croyances, représentations partagées, culturelles ou idiosyncrasiques, savoirs scolaires anciens, prescriptions, plans et procédures ; à quoi s'ajoutent, au même niveau de légitimité, les savoirs expérientiels construits par le professionnel/clinicien dans une familiarité longue vécue avec l'objet à connaître. En termes neurophysiologiques, on évoquera les frayages durablement opérés dans la masse neuronale par les expériences passées. La surprise créant incompréhension et insécurité devant l'inattendu peut être ressentie physiquement sous la forme d'un malaise. Elle pose question au travailleur surpris qui se trouve placé alors en situation de résolution de problème. Un problème se définissant par l'absence d'un mode opératoire préalable de résolution, l'apprenti ou tout opérateur dont l'attention aura été attirée par la surprise, devra résoudre le problème que lui pose la résistance que le réel oppose à la prévisibilité définie en extériorité. Pour exprimer cette indiscipline du réel, les opérateurs disent généralement : "J'ai tout de suite vu que c'était pas normal », ce qui suppose que l'opérateur ait en tête un modèle mental, une image opérative, représentation de ce qui aurait été considéré comme normal, mais aussi qu'il soit suffisamment attentif à ce qu'il perçoit dans le monde extérieur et à ce qui l'affecte dans son monde intérieur. C'est le corps sensoriel du travailleur qui devient instrument de connaissance. 
Du point de vue de la formation professionnelle, particulièrement dans les dispositifs en alternance (Mayen, 2012), il est possible d'imaginer une construction des horizons d'attente des apprentis entre :

$1 /$ des enseignements dits théoriques (pour une fois bien nommés car situés à un certain niveau de généralité et donc de prétention à la prévisibilité), savoirs de la norme diffusés par des professeurs formés à l'analyse du travail, c'est-à-dire débarrassés de l'illusion hégémonique que ce qu'ils enseignent va être mis en application sur les terrains de l'action réelle ;

2/ des « savoirs issus de la pratique », d'origine expérientielle que des formateurs aguerris pourraient, ex post, aider les agents à conscientiser, formaliser et mettre en patrimoine en leur proposant et en animant des dispositifs d'analyse de l'expérience vécue, destinés à aider les apprenants/acteurs à mettre en mots leurs savoirs d'action et à en dégager des invariants; dispositifs variés mais reposant tous sur des mises en mots, des productions langagières dialogiques et des prises de conscience susceptibles de faire apparaître des conflits moteurs de développement, entre croyances antécédentes et constats du réel de l'activité et de son contexte ${ }^{5}$.

De cette coopération entre les deux catégories de savoirs et d'acteurs si souvent en tension dans les dispositifs en alternance, on peut entrevoir une germination croisée des concepts scientifiques et des concepts quotidiens pour reprendre la formulation de Lev Vygotski (1985). L'heuristique du regard, la pédagogie de l'étonnement pourra se révéler utile pour impulser la mise en intrigue des évènements vécus par l'apprenti (Jobert et Thievenaz, 2014). Une pédagogie de l'attente et de l'écart pourrait ainsi donner plus de consistance à certaines séances indigentes, inutiles et démotivantes, de « débriefings » lors des retours de stage.

\footnotetext{
${ }^{5}$ Sur la question de la mise en mots, voir par exemple Pierre Vermersch, L'entretien d'explicitation (2019) ; ou bien l'ouvrage de Joris Thievenaz (2019).
} 


\section{Le regard didactique : regarder pour imiter}

Apprendre en regardant, regarder pour imiter et agir, sont des faits à la fois psychologiques et sociaux auxquels les anthropologues et les psychologues (en particulier chez l'enfant) s'intéressent depuis les origines de ces disciplines. S'approprier savoir-faire, conduites et valeurs d'une communauté en regardant autrui vivre et agir reste un puissant ressort de reproduction sociale et de transmission intergénérationnelle au sein de nos sociétés occidentales contemporaines développées et pas seulement dans les sociétés dites « archaïques ». Des extraits de nos carnets d'enquête en témoignent: « depuis mes six ans je venais regarder mon père à l'atelier et à son décès, j'ai repris tout naturellement sa succession »; «tout petit, je venais au magasin; caché sous une table je ne perdais rien de ce qui se passait»; "Depuis le grand père de mon père on fabrique notre fromage avec la même recette».

Comme phénomène social global, le mimétisme possède un statut ambivalent. En effet, s'il préserve, par transmission, nos sociétés du risque d'anomie, il encourage la reproduction mortifère du même et fait obstacle à l'invention d'alternatives à l'existant qui mettrait leurs auteurs au ban de leur communauté. D'autre part, il confère aux anciens qui conservent et transmettent les savoirs et valeurs légitimes des positions de pouvoir, au nom du respect des traditions ${ }^{6}$.

En revanche, les pratiques mimétiques ne sont guère mises en avant dans les formations de formateurs d'adultes professionnels, audelà de la formule «regarde-moi faire je vais te montrer»; les formateurs sont pris entre deux convictions fortes : d'une part, l'imitateur, médiocre reflet du modèle imité, ne pourra jamais prétendre à une compétence véritable; d'autre part, il est considéré comme acquis depuis la nuit des temps, que «c'est en forgeant qu'on devient forgeron» et certainement pas en regardant travailler un forgeron.

\footnotetext{
${ }^{6}$ Ainsi Emmanuel Bourdieu (1998) développe la thèse selon laquelle nos croyances ne sont pas des libres choix d'un esprit rationnel mais des dispositions, acquises par la force de l'habitude.
} 
Le mimétisme est reconnu, au plan anthropologique, comme un processus majeur d'hominisation, comme il l'est dans toutes les psychologies du développement, par Piaget comme par Vygotski, ce que chaque parent peut aussi observer quotidiennement. En revanche, pour le sens commun, le scepticisme est de mise au plan théorique en raison du caractère profondément énigmatique et de la complexité d'une prise de savoirs par le "regarder», à distance physique et interactionnelle du modèle imité et des objets sur lesquels ou avec lesquels s'accomplit l'action.

S'ajoute à cette incompréhension du processus mimétique la conception tenace d'une dissociation entre processus perceptifs et processus praxiques, lointaine rémanence dualiste qui renvoie le perceptif au biologique et l'action volontaire à une manifestation de l'esprit. Néanmoins, malgré les réticences et les ignorances, apprendre en regardant sort de sa marginalité à l'heure où, en France la loi accorde un privilège à l'apprentissage par l'action de formation en situation de travail ${ }^{7}$.

Dans la réalité du monde du travail, la pratique mimétique est très répandue dans tous les milieux, en particulier pour la formation au premier poste. En témoignent quelques citations recueillies par l'auteur sur différents terrains :

a) «Au début j'y connaissais rien, vous pensez, avant, j'avais travaillé dans le commerce, j'ai appris en regardant faire les filles; maintenant, ça va» (femme, 40 ans, importante compagnie d'assurances) ;

b) «La formation, ça ne sert à rien pour faire le travail. On nous a dit de regarder les collègues, mais elles n'avaient pas trop envie de nous montrer ; elles se cachaient pour qu'on ne voie pas » (femme, 30 ans, établissement financier de taille mondiale);

c) «Comment j'ai appris à jouer de la cornemuse? En regardant jouer les membres du groupe. Avant, je faisais la batterie » (David, musicien portugais, 32 ans);

d) «Quand j'ai été mis à ce poste, je ne connaissais rien à la conduite des ponts roulants. Avant, j'étais à la maintenance. Je suis à quinze

${ }^{7}$ Loi AFEST (Action de formation en situation de travail) du 5 septembre 2018. 
mètres de haut. Au début, j'ai regardé faire celui qui était avant moi, un type qui avait 20 ans d'expérience. Je me suis installé dans le bureau du chef qui est vitré pour mieux voir. Avec le pont, il faut faire gaffe, parce que tu passes au-dessus des gens, surtout au démarrage; au-dessus des godets en fusion avec des flammes qui sortent, avec de la fumée, de plein de machines, faut faire très gaffe, tu peux faire des catastrophes, faut avoir l'œil tout le temps c'est l'œil qui compte. Depuis trois mois, je n'ai pas fait une seule connerie, le chef ne m'a rien dit, pourtant c'est une grande gueule. Maintenant, c'est moi le pont roulant, les gens le savent » (homme, 45 ans, aciérie aciers fins).

De ces quelques citations recueillies au poste de travail (mille autres pourraient être ajoutées provenant même de secteurs d'activité à technologie complexe et à risque), on retiendra d'abord que ces apprentissages mimétiques, par le regard, ne sont pas des formations au rabais émanant d'entreprises de seconde zone cherchant le moindre coût. Si ces pratiques sont effectivement très répandues, elles forment un immense dispositif de formation professionnelle méconnu, à ciel ouvert, non formel, invisible, qui, certes, abandonne les individus à eux-mêmes, mais sont apparemment efficaces. Il faut lire et relire le petit livre de Robert Linhart, L'Établi (1978), pour y trouver, servi par une plume magnifique, le récit douloureux de certains apprentissages mimétiques abandonniques.

Au milieu des années 1990, un neurologue italien, Giacomo Rizolatti (2011), a découvert que les mêmes zones du cerveau sont activées quand on exécute soi-même une tâche et quand on l'observe exécutée par un autre. Les neurones à l'œuvre dans ce phénomène ont été appelés "neurones miroirs». Ils sont dotés de la capacité de réfléchir ce qu'ils perçoivent, au double sens du mot : ils s'approprient à l'identique ce qu'ils observent et ils interprètent le sens des informations qu'ils recueillent avant qu'elles soient mises en patrimoine par le cerveau. Le rôle des neurones miroirs est central pour l'intercompréhension humaine, jusqu'au cas limite de l'empathie qui permet de se mettre à la place de l'autre, et de faire sien ce que l'autre vit, en particulier son état émotionnel. La métaphore du miroir peut laisser penser qu'il ne s'agit que de la captation fugitive d'images venues du monde extérieur. Il n'en est rien. Les décharges neuronales 
étant identiques dans l'une et l'autre occurrence (faire ou regarder faire), leurs effets sur l'appareil neuronal sont identiques. Il y a, dans les deux cas, expérience (interaction entre neurones et ouverture de frayages neuronaux stables). Le capital mémoriel colossal dont dispose le cerveau s'en trouve modifié que l'on fasse soi-même ou que l'on regarde. On est donc bien en présence d'apprentissage.

On notera que l'étrange capacité d'appropriation par les neurones miroirs ne signifie pas qu'il suffit, pour que l'apprenti apprenne, de le laisser librement regarder faire l'expert. Encore faut-il que le novice observe ce qui est décisif dans la réussite de l'activité (ce sont souvent des détails que seul un autre expert peut percevoir). Prenons des exemples : à quoi tient dans l'intimité de mon corps en action le fait que ma balle de joueur de tennis rencontrera ma raquette, ou encore, qu'est ce qui, dans la position du corps du chanteur lui permet de faire sortir de lui le son recherché ?

Bref, l'action des neurones miroirs, ne sonne pas le glas de la fonction formative. Elle invite seulement à repérer «ce qui fait la différence » entre novice et expert. En cela l'analyse fine de l'activité nous semble nécessaire; ce qui ouvre de belles perspectives à la didactique professionnelle définie par Pastré comme analyse du travail en vue de la formation.

En formation, ce sont les deux propriétés des neurones miroirs qui sont mises à l'œuvre: l'apprenti imite pour reproduire à l'identique mais il se met aussi à la place de celui qu'il imite : double processus, l'un d'imitation, l'autre d'identification. Ce double processus, cognitif et identitaire, ne peut surprendre les formateurs expérimentés qui savent que dans toute formation professionnelle, qu'elle qu'en soit la modalité, le cognitif et l'identitaire vont et doivent aller toujours de pair. L'apprentissage est un devenir. L'heuristique de l'écart et de la surprise, propre au regard clinicien, peut suggérer bien des pratiques pédagogiques sachant qu'elles devront toutes s'affronter à la difficile mise en intrigue de la riche expérience du regard.

Pour conclure sur le regard qui apprend par mimétisme on relèvera qu'il appartient bien à la catégorie du regard qui agit puisqu'en définitive, regarder-faire produit des effets directement 
sur celui qui regarde ; regarder l'autre, m'identifier à lui me modifie. Il arrive cependant que l'inverse se produise : le regard extérieur produit des effets sur celui qui est observé ${ }^{8}$. On pense d'abord à ce qui fait la hantise des chercheurs de terrain peu expérimentés qui se demandent si leur présence ne modifie pas, voire ne fait pas disparaitre, leur objet de recherche : "Comment parlent-ils quand je ne suis pas là pour les écouter?» se demande ainsi le linguiste. Chaque chercheur est renvoyé à ses bricolages propres 9 .

Plus intéressant au chapitre de l'observation qui modifie celui qui est observé est le cas de figure où le travailleur expert, dont l'activité est observée par l'apprenti, est entraîné par l'activité de son observateur, consistant, il le sait, à comprendre ce qu'il fait. L'opérateur observé est déplacé par ce regard extérieur posé sur lui et devient lui aussi observateur de son propre travail, comme en témoigne le propos suivant recueilli auprès d'un ouvrier très qualifié du secteur de la petite mécanique : «des fois, je me demande ce qu'il (l'apprenti) est en train de regarder, ce qu'il comprend, ce qu'il en pense. Ça me fait voir autrement mon boulot, me poser des questions et c'est intéressant parce que sinon on n'a jamais le temps ». L'activité de l'observateur orientée vers l'activité de l'opérateur déplace celui-ci, entraîne une activité réflexive nouvelle sur son activité productive. Il ne fait pas de doute que ce nouveau regard porté sur soi est générateur d'un développement potentiel de son action future.

\section{Le regard praxique}

Nous avons vu successivement la perception visuelle rendre le cerveau capable d'une grande intelligence des situations, le doter d'un pouvoir d'anticipation de l'action et même le pouvoir d'inclure l'anticipation de l'action dans le processus même de la perception, de prendre en compte les conséquences de l'action sur soi-même, autrui et l'environnement, d'être disponible pour apprendre par mimétisme.

\footnotetext{
${ }^{8}$ D'où les interrogations sur l'observation participante. Voir à ce sujet la postface d'Olivier Schwartz à l'ouvrage de Nels Anderson publié en France en 1993.

${ }^{9}$ La recherche est elle-même une activité (cf. Barbier et Durand, 2015).
} 
Une dernière question sera maintenant examinée : est-ce que la remise en question de la dissociation perception/cognition/action peut-elle aller jusqu'à concevoir que le regard puisse, par lui-même, être instrument d'une action transformatrice des objets regardés. Peutil avoir une capacité performative? Si la question est oiseuse s'agissant d'objets du monde naturel, elle se pose lorsque les objets regardés sont d'autres humains, en particulier dans ce que l'on appelle les métiers de la relation (éducation, soin, service aux personnes, etc.). Par son exercice, le regard peut-il être instrument psychologique, au sens de Vygotski (1985), sorte d'appareil moteur immatériel du professionnel de la relation. Deux champs d'exercice de cette praticité du visuel seront évoqués : celui de l'éducation et celui de la formation professionnelle.

En éducation, les travaux du psychologue américain Robert Rosenthal sont éclairants. Rappelons-les succinctement. Dans une école, le chercheur fait passer à tous les élèves des tests de connaissances et de capacités dont il tire deux listes nominatives: l'une des «bons" élèves, l'autre des «mauvais». Après quoi, il ajoute quelques «bons» dans la liste des « mauvais », et inversement, avant de les remettre, sans commentaires, aux enseignants. Revenant enquêter ultérieurement, il administre à nouveau des batteries de tests. Ce qu'il constate est surprenant: les élèves étiquetés «bons » (qu'ils l'aient été réellement ou qu'ils aient été des transfuges de la liste des « mauvais ») ont tous progressé. Parallèlement, les «mauvais » (et donc aussi les transfuges de la liste des «bons ») n'ont pas progressé et certains ont régressé. C'est Pygmalion in the classroom, pour reprendre le titre de l'ouvrage de Rosenthal et Jacobson (1968). Que s'est-il passé ? Les éducateurs, informés par les chercheurs, ont reflété par leur façon de regarder les enfants, de manière non délibérée mais perceptible par les neurones miroirs, l'image normative qu'ils avaient de chacun d'eux. Cet étiquetage des enfants, porteur d'un jugement, est spontané et inévitable; on le rencontre dans les pratiques éducatives les plus ordinaires. Il est présent dans les relations entre parents et enfants, éducateurs et élèves, soignants et patients, et plus généralement dans les interactions humaines comme l'ont montré les sociologues du courant dit de l'interactionnisme symbolique. 
Si cette praticité puissante du regard est énigmatique au plan scientifique, elle n'est pas problématique au plan empirique: dans tous les lieux d'éducation, de soins aux enfants, en crèches, dans les institutions de formation des adultes, dans les milieux de travail, au sein de la famille, l'effet Rosenthal est présent, actif, constructif ou destructif. Il alimente, chez les êtres sociaux que nous sommes, un circuit social invisible de co-construction réciproque des individus au sein de communautés restreintes. Des mouvements d'éducation ont pour fondement le constat que les enfants ont tendance à se conformer à l'image d'eux-mêmes que leur renvoient les regards d'autrui portés sur eux, ce que la sagesse populaire exprime en disant: "si tu le regardes comme un incapable il le deviendra », redoutable performativité du regard.

En formation professionnelle, nous avons insisté sur l'apprentissage mimétique parce que le regard y est maître, mais aussi parce que son importance quantitative et son efficacité sont sousestimées. Il ne faudrait pas pour autant que soient également sousestimées les difficultés que pose ce triomphe du regard dans les apprentissages professionnels, soit pour des novices en apprentissage initial, soit pour des adultes occupant un poste pour la première fois, soit encore pour des professionnels en quête de façons différentes de réaliser les mêmes gestes de métier afin de construire, à partir de la diversité des « usages de soi » (Schwartz, 1987) des gens de métier qui les entourent, leur manière propre d'exercer les automatismes partagés par les hommes de métier. Yves Clot, et al. (2007) pointent que la réalisation d'une transmission imité/imitateur demande aux protagonistes de bien grandes vertus. Pour rendre compte des fondements de ce pacte exigeant entre expert et novice, le chercheur se demande avec quels outils conceptuels il est possible de théoriser ce phénomène éminemment anthropologique de la transmission transgénérationnelle des savoirs par reproduction mimétique.

Il nous semble que le phénomène peut être éclairé par le modèle ternaire du don/contredon proposé par l'anthropologue Marcel Mauss (1950). Ce modèle circulaire se compose de trois éléments liés : donner, recevoir, rendre. Au premier terme du circuit (ou le donner, le prendre et le vol consenti sont confondus), ce qui est donné 
par l'expert donateur que le novice a choisi d'observer n'est pas mince : en effet, se donner à voir, en activité, en situation réelle de production, c'est mettre en visibilité son travail réel, son engagement subjectif, ses manières propres de faire, ses transgressions, ses hésitations et ses échecs. Le second terme du circuit (recevoir), contrairement à l'idée commune, est aussi plein d'embûches. Il y faut la manière. Le circuit maussien est fragile et obéit à des règles qu'il convient de respecter pour que se prolonge le lien social. Le troisième terme du circuit maussien (rendre) appliqué à l'apprentissage mimétique semble empiriquement plus problématique : que reçoit, in fine, le donateur initial dans une transaction dont il faut rappeler qu'elle n'est pas marchande et ne fonctionne pas à l'équivalence monétaire ? Dans le modèle canonique, c'est parce que le donateur initial peut devenir à son tour donataire que le lien social se perpétue. Les tuteurs disent que le cycle est accomplit lorsque celui qui apprend a bien pris ce qui a été donné, qu'il est devenu un " pro » à son tour, et qu'il a manifesté sa gratitude.

Á l'occasion de nos travaux de terrain, nous avons observé deux façons dont le donateur initiatique peut trouver son compte (symbolique) à avoir consenti à être regardé, et donc une motivation à entretenir le lien.

Dans la première, l'imitateur va reproduire, dans l'espace social de travail ouvert, la manière propre d'agir du modèle observé. C'est une façon pour le modèle de déployer dans un espace élargi et de rendre visible le savoir-faire et le savoir-se-conduire (normes techniques et éthiques toujours mêlées) qui le définissent identitairement et d'en tirer reconnaissance: «Toi, (dira-t-on à l'apprenti devenu opérateur), on voit tout de suite qui t'a montré le boulot $\gg$. Façon aussi pour le modèle de se prolonger dans le temps, à travers un apprenti encore jeune, au-delà de son temps actif de travail et peut-être au-delà de la mort ("je veux transmettre ce que je sais faire pour que le métier ne se perde pas quand les anciens seront partis »). En recevant le don et en le remettant en quelque sorte en circulation dans l'espace social, l'apprenti a détaché du modèle l'action imitée et, par là même, lui a donné le statut d'une œuvre, selon les catégories d'Hannah Arendt (1983). Cette mise en circulation de l'esprit du modèle imité (son «âme», dit Mauss) lui confère une 
durabilité qui prolonge dans le temps, au-delà de la personne du modèle, la mémoire de lui-même son âme continue à vivre pour témoigner de son action passée.

Dans la seconde façon, ce qui est rendu au modèle observé peut prendre une autre forme, qui illustre le fort potentiel praxique du regard. Tout se passe comme si le fait d'être regardé en train d'agir déplaçait le regard que le modèle observé pose sur ses propres actes et l'entraînait à devenir l'observateur de sa propre activité et de luimême agissant, comme l'indique la remarque de l'opérateur cité cidessus : "Des fois je me demande ce qu'il (l'apprenti) regarde, ce qu'il ne comprend pas; ça me fait voir autrement comment je fais, C'est intéressant parce que sinon, on n'a jamais le temps » (ouvrier hautement qualifié, grosse mécanique). Il ne fait pas de doute que ce déplacement du regard porté par l'opérateur sur lui-même est source de développement potentiel de son action.

\section{Conclusion : un nouveau regard sur l'ingénierie de la formation professionnelle couplée à la recherche}

Regard herméneute, didactique, praxique, la formation professionnelle pourrait-elle ainsi se définir comme éducation d'un regard pour l'action, centré sur les relations qui s'établissent entre un corps qui perçoit, souffre, retient, désire, et pense, et le monde dans lequel et sur lequel il agit ou se prépare à agir. Une telle perspective exige en préalable une rupture radicale avec toute forme de dualisme, qui inspire encore la vision d'une formation de l'esprit en vue de le préparer à gouverner de manière normée un corps moteur au service de l'agir professionnel.

\section{Références bibliographiques}

ARENDT H. (1983), Condition de l'homme moderne, [1958], Paris, Éditions Calmann-Lévy.

BACHELARD G. (1971), La formation de l'esprit scientifique, [1938], Paris, Librairie Vrin. 
BARBIER J-M., DURAND M. (dir.), (2015), Sujets, activités, environnements, Paris, Presses Universitaires de France.

BERTHOZ A. (1997), Le sens du mouvement, Paris, Éditions Odile Jacob.

BOURDIEU E. (1998), Savoir-faire. Contribution à une théorie dispositionnelle de l'action, Paris, Éditions du Seuil.

CLOT Y., FERNANDEZ G. et SCHELLER L. (2007), « Le geste de métier: problèmes de transmission", Psychologie de l'interaction, $\mathrm{n}^{\circ} 8$, p. 111-126.

CLOT Y. (1995), Le travail sans l'homme? Pour une psychologie des milieux de travail et de vie, Paris, Éditions La Découverte.

DEPRAZ N., VARELA F.-J. et VERMERSCH P. (2011), Á l'épreuve de l'expérience. Pour une pratique phénoménologique, Bucarest, Zeta Books.

ECO U. (1992), Le signe: histoire et analyse d'un concept, Paris, Livre de Poche.

HOUDÉ O. (2018), L'école du cerveau: De Montessori, Freinet et Piaget aux sciences cognitives, Paris, Éditions Mardaga.

JOBERT G. (2006), «Entre savoirs et surprise, une analyse clinique du travail », dans Cifali M. et Giust Desprairies F., De la clinique : Un engagement pour la formation et la recherche, Bruxelles, Éditions de Boeck.

JOBERT G. (2014), Exister au travail. Les hommes du nucléaire, Toulouse, Éditions Érès.

JOBERT G. et THIEVENAZ J. (2014), «L'homo demirans ou l'homme qui, s'étonnant, devient connaissant», Éducation permanente, $\mathrm{n}^{\circ} 200$.

LHUILLIER D. (2006), Cliniques du travail, Toulouse, Éditions Érès. LINHART R. (1978), L’Établi, Paris, Éditions de Minuit. 
MARCELLI D. (2006), La surprise : chatouille de l'âme, Paris, Albin Michel.

MAUSS M. (2003), Sociologie et anthropologie, [1934], Paris, Presses Universitaires de France, p. 145-279.

MAYEN P. (2012), «Questions d'apprentissage dans les formations en alternance », Éducation permanente, $\mathrm{n}^{\circ} 193$.

MERLEAU-PONTY M. (1952), Phénoménologie de la perception, [1945], Paris, Éditions Gallimard.

NACCACHE L. (2006), Le nouvel inconscient. Freud, Christophe Colomb des neurosciences, Paris, Éditions Odile Jacob.

OCHANINE D.-A. (1971), «L'acte et l'image, problème d'ergonomie ", Actes du XVII ème Congrès, Association internationale de psychologie appliquée, Bruxelles, p. 81-89.

PASTRÉ P. (2011), La didactique professionnelle: approche anthropologique du développement chez les adultes, Paris, Presses Universitaires de France.

RICOEUR P. (1998), Du texte à l'action. Essais d'herméneutique, Paris, Éditions du Seuil, Coll. Points.

RIZZOLATTI G., SINIGAGLIA C. (2011), Les neurones miroirs, Paris, Éditions Odile Jacob.

ROSENTHAL R., JACOBSON L. (1968), Pygmalion in the Classroom, Holt, Reinhart and Winston.

SCHWARTZ O. (1993), "L'empirisme irréductible», dans N. Anderson, Le Hobo. Sociologie du sans-abri, [1923], Paris, Éditions Nathan, p. 265-308.

SCHWARTZ Y. (1987), "Travail et usage de soi », dans M. Bertrand et Ali., Je, Sur l'individualité, Paris, Éditions Sociales, p. 181-207.

SCHWARTZ Y. (1988), Expérience et connaissance du travail, Paris, Messidor/Éditions Sociales. 
THIEVENAZ J. (2019), Enquêter et apprendre au travail : Approcher l'expérience avec John Dewey, Dijon, Éditions Raison et Passions.

VARELA F.-J., THOMPSON E. et ROSCH E. (1993), L'inscription corporelle de l'esprit: sciences cognitives et expérience humaine, Paris, Éditions du Seuil.

VERMERSCH P. (2019), L'entretien d'explicitation, Paris, ESF Sciences Humaines.

VYGOTSKI L.-S. (1985), Pensée et langage, [1934], Paris, Éditions Sociales. 